\title{
220. Operativ-technische Maßnahmen zur Vermeidung einer Insuffizienz bei Dickdarm- insbesondere Rectumresektionen
}

\author{
A. Andriopoulos, P. Wirsing und R. Bötticher \\ Chirurgische Klinik I, Stadtkrankenhaus, D-8510 Fürth
}

\section{Technical Operative Procedures to Avoid Leakage of Anastomoses of the Colon and Rectum}

Summary. We report on 331 colon resections. The postoperative mortality due to leakage of the anastomosis was $1.7 \%$. Dehiscence can be caused by bacterial infections, disturbances of microcirculation, catabolism, clotting disorders and immunological defects. Meticulous dissection of the bowel and the suture technique is of great importance to avoid insufficiency. We recommend the following procedure: Preoperative orthograde irrigation; perioperative antibiotic prophylaxis (mezlocillin-metronidazol); minimally dissected margins of the bowel stumps; cutting with scissors or scalpel, no electrocoalgulation; preservation of the marginal arcade; extramucosal suture technique with absorbable material; absence of tension; eventual use of fibrin adhesive human liquid; retroperitonealization and drainage of the anastomosis with Penrose in deep resection of the rectum.

Key words: Colon anastomosis - Leakage avoidance.

Zusammenfassung. Wir berichten über 331 Colonresektionen. Die Letalität wegen einer Anastomoseninsuffizienz betrug 1,7\%. Obwohl Nahtdehiscenzen durch bakterielle Infektion, Störung der Mikrozirkulation, Katabolie, Gerinnungsstörungen, Tumorkrankheit, Immundefekte mitverursacht werden, nimmt die Nahttechnik nebst entsprechender Präparation des zu resezierenden Darmes eine zentrale Stellung ein. Wir streben folgendes Vorgehen an: Prograde präoperative Darmspülung. Perioperative Antibioticaprophylaxe (Mezlocillin-Metronidazol). Offene Resektion mit sparsamer Skelettierung an den Resektionsrändern. Durchtrennung mit Schere oder Messer (keine Diathermie). Erhaltung der Randarkade. Extramucöse Nahttechnik in 4-5 mm Abstand mit resorbierbarem Material. Eventuell Kleben der Anastomose mit Fïbrinkleber bei intraperitonealer Verbindung. Retroperitonealisierung und Drainage mit Penrose bei tiefen Rectumresektionen.

Schliisselwörter: Dickdarmanastomose - Insuffizienzvermeidung.

\section{Das Risiko in der Colonchirurgie bei über 80jährigen Patienten}

\author{
L. C. Tung, R. Häring, G. Görtz und U. Kania
}

Chirurgische Klinik und Poliklinik im Klinikum Steglitz der Freien Universität Berlin, Hindenburgdamm 30, D-1000 Berlin 45

\section{Risk of Colon Surgery in Patients over 80 Years Old}

Summary. A total of 120 patients over 80 years of age with colorectal carcinoma were treated. Over $90 \%$ were admitted 4.5 months after typical symptoms occurred. The carcinoma was located at the rectosigmoid in $60 \%$ and was found in an advanced stage in $98 \%$. Emergency operations were carried out on 25 patients (mortality: $48 \%$ ) and elective operations on 95 patients (mortality: $30.5 \%$ ). On average each had 3.3 accompanying diseases. A total of 76 patients were discharged. The survival rate at 1 year was $55 \%, 2$ years $46 \%$ and 3 years $28 \%$. The longest survival was 12 years.

Key words: Colorectal carcinoma - Advanced age.

Zusammenfassung. 120 über 80jährige Patienten mit colorectalem Carcinom wurden behandelt. Die Klinikaufnahme erfolgte bei über $90 \%$ der Patienten $41 / 2$ Monate nach Auftreten typischer Symptome. Das Carcinom lag in $60 \%$ im Rectosigmoid und war in $98 \%$ in fortgeschrittenem Stadium. Noteingriff wurde bei 25 Patienten (Letalität: $48 \%$ ) und Elektiveingriff bei 95 Patienten (Letalität: $30,5 \%$ ) durchgeführt. Durchschnittlich hatte jeder Patient 3,3 Begleiterkrankungen. 76 Patienten wurden entlassen: Überlebensrate nach einem Jahr 55\%, nach 2 Jahren $46 \%$ und nach 3 Jahren $28 \%$. Die längste Überlebenszeit war 12 Jahre.

Schliusselwörter: Alterschirurgie - Colorectales Carcinom. 